\title{
Accidental Intrathecal Administration of Tranexamic Acid: A Case Report
}

\author{
Karl Matthew C Sy Su MD* \\ Department of Anesthesiology, Philippine General Hospital, University of the Philippines Manila, Philippines
}

\begin{abstract}
Medication errors are still one of the contributing factors leading to morbidity and mortality in anesthesia, despite measures to ensure patient safety. A 14-year-old male inadvertently received intrathecal tranexamic acid instead of hyperbaric bupivacaine for an elective herniorrhaphy. Shortly after induction, patient complained of severe back and lower limb pain, restlessness, tachycardia, hypertension, and generalized myoclonic seizures. Cardiopulmonary support was immediately instituted, and convulsions were managed by intravenous midazolam, diazepam, and phenytoin, and induction of general anesthesia. Cerebrospinal lavage was also performed to reduce and dilute amounts of injected drug in the cerebrospinal fluid. The surgery was temporarily deferred with the patient's condition progressively improved to full recovery without neurologic sequelae. The error was attributed to similarities in appearance between ampules of tranexamic acid and hyperbaric bupivacaine. This report highlights the importance of double-checking medications prior to administration to avoid medication errors and ensure patient safety.
\end{abstract}

\section{Keywords}

Medication errors, Spinal anesthesia, Tranexamic acid

\section{Introduction}

Medication errors continue to be a leading cause of morbidity and mortality even in industrialized countries, despite vigilance in anesthesia practice. Several factors have been previously identified as contributory. These include wrong labeling, incorrect location, or similar appearance of ampules and syringes; human errors such as inattention, poor communication, and fatigue; and lack of systematic medication safety guidelines and practices. We report the accidental injection of tranexamic acid (TXA) into the subarachnoid space due to similarity in appearance between the ampules of the drug and hyperbaric bupivacaine for spinal anesthesia, leading to severe pain in the back and both lower limbs, hypertension, tachycardia, and generalized myoclonic seizures.

\section{Case summary}

A 14-year old previously healthy male was scheduled for an elective right herniorrhaphy at a primary hospital under spinal anesthesia. Lumbar puncture was aseptically performed in the left lateral decubitus position, using a 27-gauge Quincke needle at the L3-4 interspace, with $3 \mathrm{~mL}$ of solution being injected intrathecally. Three minutes after the patient was placed in the supine position, he became restless and complained of severe pain in both lower limbs and back. His pulse rate and blood pressure increased to 130 bpm and 160/100 $\mathrm{mmHg}$, respectively. Intravenous sedation with midazolam ( $3 \mathrm{mg}$ ) and propofol $(100 \mathrm{mg}$ ) along with intravenous labetalol $(5 \mathrm{mg}$ ) to control heart rate and blood pressure was administered. A decision to secure his airway through endotracheal intubation was made and he was then maintained on mechanical ventilation with $100 \%$ oxygen and sevoflurane $3 \%$ by volume. The surgery was then postponed.

A rapid survey of previously administered medications revealed tranexamic acid (300 $\mathrm{mg}$ ) was accidentally administered into the subarachnoid space instead of $15 \mathrm{mg}$ of hyperbaric bupivacaine. To decrease the concentration of the drug in the cerebrospinal fluid (CSF), CSF lavage with aliquots of normal saline (total volume of $120 \mathrm{~mL}$ ) was performed on the patient with an epidural catheter inserted at the L3-4 subarachnoid space. Close observation and continuous sedation were instituted with the vital signs of the patient maintained within acceptable limits.

*Corresponding author: Karl Matthew C Sy Su MD, Department of Anesthesiology, Philippine General Hospital, University of the Philippines Manila, Philippines

Accepted: July 21, 2021

Published online: July 23, 2021

Citation: Sy Su, KMC (2021) Accidental Intrathecal Administration of Tranexamic Acid: A Case Report. J Clin Anesth Pain Manag $5(2): 232-234$ 
An hour after intrathecal administration of the wrong drug, the patient developed generalized myoclonic seizures. An intravenous dose of propofol $40 \mathrm{mg}$ was given together with a loading dose of mannitol $40 \mathrm{~g}$ followed by $20 \mathrm{~g}$ every eight hours. However, multiple seizure episodes persisted, prompting administration of intermittent propofol boluses, and intravenous diazepam and dexamethasone $(8 \mathrm{mg})$ at regular intervals. Eight hours from the onset of generalized myoclonic seizures, a loading dose of phenytoin $(15 \mathrm{mg} / \mathrm{kg})$ was given over 14 minutes with no further seizures noted.

The patient was weaned from sedation nine hours after the incident, with improvement in the patient's consciousness and vital parameters. However, there was persistence of back pain that was relieved by intravenous analgesics. Twenty hours after the administration of the wrong drug, the patient was extubated fully awake, able to follow commands, and had no seizure recurrence. After consultation with a pediatrician, oral phenobarbital $200 \mathrm{mg}$ daily for one month was initiated. The patient was subsequently discharged after two weeks of hospital admission with no neurological deficits.

\section{Discussion}

Tranexamic acid [4-(aminomethyl) cyclohexanecarboxylic acid] is a synthetic lysine analog that shows high affinity for the lysine binding sites of plasminogen and plasmin, explaining its role in preventing bleeding. It is considered safe and affordable with few serious adverse effects reported; hence, it is widely used in the control of traumatic, surgical, and obstetric hemorrhage. However, neurotoxicity and seizures have been reported in animal studies. There is furthermore a paucity of data available on the effects of TXA when administered through direct intrathecal injection [1].

Wong and colleagues first reported a case of an 18-yearold male for appendectomy who was injected with TXA 75 $\mathrm{mg}$ intrathecally. This resulted in generalized seizures treated with intravenous diazepam, with the patient recovering completely without deficit [2]. Since then, there have been 21 identified reports of accidental subarachnoid administration of TXA [3]. Typical signs and symptoms of intrathecal TXA injection include the absence of sensorimotor block; severe pain in the back, buttocks, and legs; myoclonus to generalized convulsions; tachycardia and hypertension; and ventricular arrhythmia. Death was reported in 10 of these patients, thus placing the mortality rate at close to $50 \%$ [3]. Tranexamic acid at a therapeutic dose of $1 \mathrm{~g}$ intravenously will result in a CSF concentration of $0.9 \mathrm{mcg} / \mathrm{L} 9$ hours after administration; the injection of a similar dose through a normal CSF volume would be expected to produce a concentration in the CSF 7000 times higher or approximately $6,600 \mathrm{mcg} / \mathrm{mL}$. The administration of $200 \mathrm{mg}$ TXA in a patient who subsequently died yielded a post-mortem CSF TXA concentration of $432 \mathrm{mcg} / \mathrm{mL}$ [4].

The mechanisms for subarachnoid TXA-induced symptoms are still under investigation. Immediate back and gluteal pain and leg myoclonus may be secondary to an increase in the excitability of neuronal networks by directly inhibiting $\gamma$-aminobutyric acid type $A$ and glycine receptors found on the postsynaptic sites of spinal dorsal horn neurons on top of a direct local effect on the spinal cord dorsal horn [5]. Generalized convulsions may be due to decreased inhibitory neurotransmission resulting from the direct competitive antagonism of cerebral $\mathrm{\gamma}$-aminobutyric acid type $\mathrm{A}$ and glycine receptors, compounded by cerebral vasoconstriction, ischemia, and increased intracranial pressure [6]. The seizure activity may further result in cardiovascular effects through a centrally mediated sympathetic activization.

Management of inadvertent intrathecal TXA administration is primarily symptomatic and supportive. Multiple anticonvulsants may be necessary to terminate seizures, with some authors suggesting thiopental infusion while on electroencephalographic monitoring [7]. Magnesium sulfate has also been demonstrated to be effective in terminating status epilepticus [8]. Intravenous phenytoin and oral phenobarbital were used in our patient due to their availability in the primary hospital. Several general anesthetics, including the inhalational agent sevoflurane, and the intravenous anesthetic propofol, were likewise used in the termination of the seizures for their action as positive allosteric modulators of glycine receptors [6].

Toxic effects of intrathecal tranexamic acid may be reduced by reducing its CSF concentration. CSF lavage, as was done in this patient, removes and dilutes the injected TXA, thereby preventing the possibility of further neuronal excitotoxic damage. This is performed by initial aspiration of CSF and repeated replacement with normal saline in small volumes, while maintaining careful asepsis. The treatment of convulsions and CSF lavage when performed earlier lead to favorable outcomes [9]. Some authors suggest concomitant or subsequent administration of local anesthetic as a viable alternative [2]. Seizures and arrhythmias may persist or recur for days suggesting a slow decrease in CSF and neuronal levels of TXA following intrathecal administration. Cardiorespiratory support should then be maintained. Labetalol was also administered to this patient to control tachycardia and hypertension. Prophylactic amiodarone has also been reported in one case to prevent arrhythmias and ventricular fibrillation [7].

Finally, the accidental administration occurred due to confusion between TXA and hyperbaric bupivacaine because of the similarity of appearance of the ampules (Figure 1). Several measures should be undertaken in the prevention of medication errors during neuraxial anesthesia including careful reading of clear drug labels, and separating storage of TXA and other non-anesthetic drugs from local anesthetics intended for subarachnoid administration.

\section{Conclusion}

In conclusion, cases of inadvertent intrathecal administration of TXA were due to confusion between the similar appearances of the ampules of TXA and hyperbaric bupivacaine. Human errors resulting from time constraints, unfamiliar setting, and fatigue are also contributory. Preventive strategies including clear labeling, proper storage, and a systematic approach to ensure accurate identification of medications are integral components of programs to ensure patient safety. There is no definitive treatment protocol for intrathecal TXA administration; however, using the cornerstones of intensive hemodynamic monitoring, cardiorespiratory support, anticonvulsant therapy, and CSF lavage may be key in the prevention of catastrophic results. 


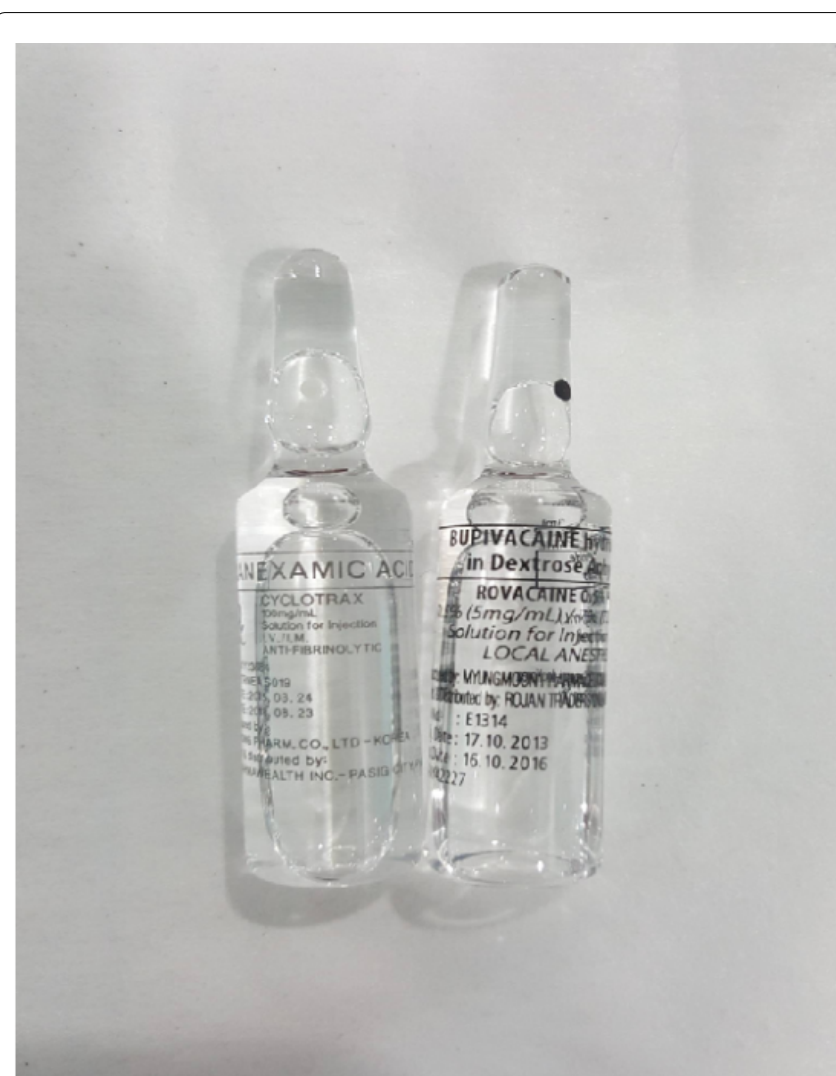

Figure 1: Ampules of tranexamic acid (left) and hyperbaric bupivacaine $0.5 \%$ (right).

\section{References}

1. Mahmoud K, Ammar A (2012) Accidental injection of tranexamic acid. Case Reports in Anesthesiology 2012: 646028.

2. Wong JO, Yang SF, Tsai MH (1988) Accidental injection of tranexamic acid (Trasamin) during spinal anesthesia. Anaesthesiologica Sinica 26: 249-252.

3. Patel S, Robertson B, McConachie I (2019) Catastrophic drug errors involving tranexamic acid administered during spinal anesthesia. Anaesthesia 74: 904-914.

4. Kim MJ, Jeong SJ, Choi ES, et al. (2009) Death after accidental injection of tranexamic acid during spinal anesthesia. Korean Journal of Legal Medicine 33: 139-142.

5. Ohashi N, Ohashi M, Endo N, et al. (2017) Administration of tranexamic acid to patients undergoing surgery for adolescent idiopathioc scoliosis evokes pain and increases the infusion rate of remifentai during the surgery. PLoS ONE 12: e013622.

6. Lecker I, Wang DS, Whissell PD, et al. (2016) Tranexamic acidassociated seizures: Causes and treatment. Ann Neurol 79: 18-26.

7. Butala BP, Shah VR, Bhosale GP, et al. (2012) Medication error. Subarachnoid injection of tranexamic acid. Indian J Anaesth 56: 168-170.

8. Hatch DM, Atito-Narh E, Herschmiller EJ, et al. (2016) Refractory status epilepticus after inadvertent spinal injection of tranexamic acid treated by magnesium sulfate. Int J Obstet Anesth 26: 71-75.

9. Narra GR (2015) Accidental injection of tranexamic acid into intrathecal space. Journal of Research in Anaesthesiology and Pain Medicine 4: 1910-1916.

DOI: $10.36959 / 377 / 359$

Copyright: (C) 2021 Sy Su, KMC. This is an open-access article distributed under the terms of the Creative Commons Attribution License, which permits unrestricted use, distribution, and reproduction in any medium, provided the original author and source are credited. 